\title{
Dynamic Manipulability of Multifingered Grasping
}

\section{$\operatorname{AUTHOR}(S)$ :}

Yokokohji, Yasuyoshi; Martin, Jose San; Fujiwara, Masaki

\section{CITATION:}

Yokokohji, Yasuyoshi ...[et al]. Dynamic Manipulability of Multifingered Grasping. IEEE TRANSACTIONS ON ROBOTICS 2009, 25(4): 947-954

\section{ISSUE DATE:}

2009-08

URL:

http://hdl.handle.net/2433/109807

\section{RIGHT:}

(c) 2009 IEEE. Personal use of this material is permitted. However, permission to reprint/republish this material for advertising or promotional purposes or for creating new collective works for resale or redistribution to servers or lists, or to reuse any copyrighted component of this work in other works must be obtained from the IEEE. 
[37] S. Ekvall and D. Kragic, "Learning and evaluation of the approach vector for automatic grasp generation and planning," in Proc. IEEE Int. Conf. Robot. Autom., Roma, Italy, Apr. 10-14, 2007, pp. 4715-4720.

[38] W. Yao and J. S. Dai, "Dexterous manipulation of origami cartons with robotic fingers based on the interactive configuration space," $A S M E$ Trans.: J. Mech. Design, vol. 130, no. 2, pp. 022303-1-022303-8, 2008.

[39] E. Rodriguez-Leal and J. S. Dai, "From Origami to a new class of centralized 3-DOF parallel mechanisms, DETC2007-35516," presented at the 31st ASME Mechanisms Robot. Conf., ASME Int. Design Eng. Tech. Conf. Comput. Inform. Eng. Conf., Las Vegas, NV, Sep 4-7, 2007.

\section{Dynamic Manipulability of Multifingered Grasping}

Yasuyoshi Yokokohji, Jose San Martin, and Masaki Fujiwara

\begin{abstract}
In this paper, we extend the concept of dynamic manipulability to evaluate the dynamic property of multifingered grasping systems consisting of a multifingered hand and a grasped object, and propose a measure of dynamic manipulability of multifingered grasping. Similar to the original dynamic manipulability, the proposed measure evaluates the mapping from a set of realizable joint torques to a set of resultant accelerations of the grasped object, which forms an ellipsoid under a constant internal force constraint. It is clearly shown that the internal forces not only affect the volume of the ellipsoid, but also the amount of offset of the ellipsoid, while the gravity forces simply induce an offset. A new measure, i.e., omnidirectionality, is introduced to add a penalty to the original manipulability measure, which simply evaluates the volume of the ellipsoid, depending on how much the ellipsoid is offset. Numerical examples by using a simple two-fingered robot hand are shown to demonstrate the effectiveness of the proposed measure.
\end{abstract}

Index Terms-Dynamic manipulability, grasping, internal force, multifingered hand, omnidirectionality.

\section{INTRODUCTION}

Dynamic manipulability was originally proposed as a measure of dynamic property for single manipulators [17] ${ }^{1}$ In this paper, we extend the concept of dynamic manipulability to evaluate the dynamic property of multifingered grasping systems consisting of a multifingered hand and a grasped object. In a sense, each finger of a multifingered robotic hand can be regarded as a small manipulator. However, we cannot simply apply the measure for single manipulators to each finger of the hand, because each finger does not move independently in most cases, rather the fingers move cooperatively. Therefore, we must consider the dynamic manipulability of multifingered systems under the kinematic constraint, including the contact model (e.g., point contact or rolling contact), internal forces (or grasping forces), and the contact condition (i.e., friction condition).

Manuscript received February 9, 2008; revised September 29, 2008. First published June 5, 2009; current version published July 31, 2009. This paper was recommended for publication by Associate Editor H. R. Choi and Editor K. Lynch upon evaluation of the reviewers' comments.

Y. Yokokohji is with the Department of Mechanical Engineering and Science, Graduate School of Engineering, Kyoto University, Kyoto 606-8501, Japan (email: yokokohji@me.kyoto-u.ac.jp).

J. S. Martin is with the Department of Computers Architecture, Universidad Rey Juan Carlos, Mostoles 28933, Spain (e-mail: jose.sanmartin@urjc.es).

M. Fujiwara was with the Department of Mechanical Engineering, Graduate School of Engineering, Kyoto University, Kyoto 606-8501, Japan. $\mathrm{He}$ is now with Keyence Corporation, Osaka 536-0002, Japan (e-mail: mskfjwr7@qj9.so-net.ne.jp).

Color versions of one or more of the figures in this paper are available online at http://ieeexplore.ieee.org.

Digital Object Identifier 10.1109/TRO.2009.2022433

${ }^{1}$ Note that the definition of dynamic manipulability ellipsoid was corrected in [19] in accordance with the new definition of dynamic manipulability ellipsoid by Chiacchio [8].
The original manipulability concept, which evaluates the mapping from joint velocity to the reference point velocity, was extended to the multiarm case by several researchers. Lee defined the dual-arm manipulability [13] and Chiacchio et al. [4] formulated the manipulability of cooperating robots in a more general way. Bicchi et al. [1] generalized the concept of manipulability for coordinated manipulation, including the case of kinematicaly defective element (i.e., lacking enough degrees of freedom), such as whole-arm manipulation and power grasping. Bicchi and Prattichizzo further extended the manipulability measure for cooperating robots with passive joints [3].

So far, just a few research works focused on the dynamic manipulability problem for coordinated robots. Chiacchio et al. extended their work [4] to task space dynamic analysis and introduced the dynamic manipulability ellipsoid for multiarm systems [5]. They also discussed the effect of gravity in [5] as they discussed for the single-arm case [6]. In their formulation, however, the effect of internal forces, which is an important aspect of multifingered grasping, was not explicitly considered. Bicchi et al. [2] formulated the dynamic manipulability as a ratio between the input joint torque and the resultant grasped object acceleration. Again, no internal forces are explicitly considered in their formulation. Zheng et al. [21] proposed a dynamic manipulability measure of multiple robotic mechanisms in coordinated manipulation. Instead of calculating the volume of the dynamic manipulability ellipsoid, they calculated a convex polyhedron as a set of feasible accelerations of the grasped object (acceleration polytope) under the joint torque limitations and friction constraints. However, calculating the convex polyhedron is computationally expensive (no analytic method), and the effect of internal forces is not clear in their method.

When a robotic hand grasps an object, certain amount of grasping forces must be applied. This internal force may sacrifice the ability to accelerate the object, since the realizable torque for each joint is limited. Then, it would be possible to define the dynamic manipulability of multifingered grasping as the set of all possible maximum accelerations of the grasped object by all possible fingertip forces under the friction condition. Unfortunately, such a set is difficult to obtain analytically. In this paper, we extend the concept of the original dynamic manipulability to evaluate the dynamic property of multifingered grasping systems consisting of a multifingered hand and a grasped object, and propose a measure of dynamic manipulability of multifingered grasping. It will be shown that by applying all possible joint torques under a constant internal force constraint, the set of resultant accelerations of the grasped object forms an ellipsoid (dynamic manipulability ellipsoid of multifingered grasping). Similar to the original dynamic manipulability measure, we can easily calculate the volume of this ellipsoid, which is defined as a measure of dynamic manipulability of multifingered grasping. Therefore, to evaluate the dynamic manipulability of a multifingered system, one has to specify certain internal forces under which all possible fingertip forces satisfy the friction condition.

The ability to apply internal forces by a robot hand can be evaluated by the internal force manipulability defined in [3] or [4]. However, the internal force manipulability in [3] and [4] is just for evaluating the mapping from the joint torque space to the resultant internal force space. What we want to evaluate is the mapping from the joint torque space to the resultant grasped object acceleration space under a certain amount of internal force.

With the proposed measure, it will be clearly shown that the internal forces not only affect the volume of the ellipsoid, but also the amount of offset of the ellipsoid, while the gravity forces simply induce an offset. A new measure, i.e., omnidirectionality, is also introduced to add a penalty to the original manipulability measure, which simply evaluates the volume of the ellipsoid, depending on how much the ellipsoid is offset. 


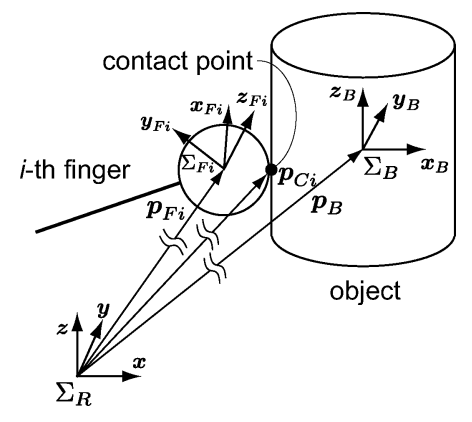

Fig. 1. Fingertip and object.

Numerical examples by using a simple two-fingered robot hand are shown to demonstrate the effectiveness of the proposed measure, including the effect of offsets of the ellipsoids.

\section{Dynamics of A Multifingered Hand System}

\section{A. Grasping Constraints}

To realize a fingertip force in any direction in 3-D space, a finger must have at least three (usually rotational) joints that are not all in parallel. Although it is possible to deal with redundant fingers, having more than three joints, we assume a robotic hand with $n(\geq 3)$ fingers, each of which has three joints to make the following formulation simple. In order to study 2-D tasks like the one shown in the numerical example in Section IV, the hand will be reduced to $n^{\prime}(\geq 2)$ fingers, each of which has just two joints.

We assume that each finger makes a frictional point contact with an object, including fixed point contact and hard rolling contact without moment around the contact normal. Although the following formulations cover rolling contact with a round fingertip, as shown Fig. 1, no essential effect of rolling contact constraint will appear because we neglect velocity terms when we discuss the dynamics of the total system. It would also be possible to deal with the soft-finger contact (or pure rolling contact) case. In such a case, each finger must have at least four joints, but the minimum number of fingers becomes just two even for 3-D space.

As shown in Fig. 1, a base coordinate frame $\Sigma_{R}$ is attached to the hand base and an object coordinate frame $\Sigma_{B}$ is placed at the gravity center of the object. Each fingertip coordinate frame $\Sigma_{F i}$ is located at the reference point of the $i$ th fingertip $(i=1, \ldots, n)$. Position and orientation of $\Sigma_{B}$ with respect to $\Sigma_{R}$ are represented by a vector $\boldsymbol{p}_{B} \in \Re^{3}$ and a rotation matrix $\boldsymbol{R}_{B} \in \Re^{3 \times 3}$, respectively. In the same way, position and orientation of $\Sigma_{F i}$ are given by $\boldsymbol{p}_{F i} \in \Re^{3}$ and $\boldsymbol{R}_{F i} \in$ $\Re^{3 \times 3}$, respectively. The $i$ th finger's contact point is denoted by $\boldsymbol{p}_{C i} \in$ $\Re^{3}$. In the case of fixed point contact, $\Sigma_{F i}$ could be located at $\boldsymbol{p}_{C i}$.

If no tangential slip occurs at the $i$ th finger's contact point, we get the following constraint between the object velocity and the fingertip velocity [9], [20]:

$$
\boldsymbol{D}_{B i}\left[\begin{array}{c}
\dot{\boldsymbol{p}}_{B} \\
\boldsymbol{\omega}_{B}
\end{array}\right]=\boldsymbol{D}_{F i}\left[\begin{array}{c}
\dot{\boldsymbol{p}}_{F i} \\
\boldsymbol{\omega}_{F i}
\end{array}\right]
$$

where $\boldsymbol{\omega}_{B} \in \Re^{3}$ and $\boldsymbol{\omega}_{F i} \in \Re^{3}$ denote angular velocities of the object and the $i$ th fingertip, respectively. Matrices $\boldsymbol{D}_{B i} \in \Re^{3 \times 6}$ and $\boldsymbol{D}_{F i} \in$ $\Re^{3 \times 6}$ are defined as follows:

$$
\begin{aligned}
& \boldsymbol{D}_{B i} \triangleq\left[\boldsymbol{I}_{3}-\left[\left(\boldsymbol{p}_{C i}-\boldsymbol{p}_{B}\right) \times\right]\right] \\
& \boldsymbol{D}_{F i} \triangleq\left[\boldsymbol{I}_{3}-\left[\left(\boldsymbol{p}_{C i}-\boldsymbol{p}_{F i}\right) \times\right]\right]
\end{aligned}
$$

where $[\cdot x]$ denotes a skew-symmetric matrix that is equivalent to the cross-product operation. Note that (1) represents the contact point velocity in the case of fixed point contact. Also note that (1) is valid for hard rolling contact as long as no tangential slippage occurs [20].

A fingertip velocity and the corresponding joint velocities are related by

$$
\left[\begin{array}{c}
\dot{\boldsymbol{p}}_{F i} \\
\boldsymbol{\omega}_{F i}
\end{array}\right]=\boldsymbol{J}_{F i} \dot{\boldsymbol{\theta}}_{i}
$$

where $\boldsymbol{\theta}_{i} \in \Re^{3}$ denotes the joint vector of the $i$ th finger and $\boldsymbol{J}_{F i} \in$ $\Re^{6 \times 3}$ is the Jacobian matrix of the $i$ th finger. From (1) and (4), we define the following Jacobian matrix:

$$
\boldsymbol{D}_{F i} \boldsymbol{J}_{F i} \dot{\boldsymbol{\theta}}_{i} \triangleq \boldsymbol{J}_{C F i} \dot{\boldsymbol{\theta}}_{i}
$$

We will represent the object velocity as a 6-D vector $\boldsymbol{v}_{B} \triangleq\left[\begin{array}{ll}\dot{\boldsymbol{p}}_{B}^{T} & \boldsymbol{\omega}_{B}^{T}\end{array}\right]^{T}$. From (1) and (5), the constraint between the joint velocity $\dot{\boldsymbol{\theta}} \triangleq \operatorname{col}\left[\dot{\boldsymbol{\theta}}_{1}, \dot{\boldsymbol{\theta}}_{2}, \ldots, \dot{\boldsymbol{\theta}}_{n}\right] \in \Re^{3 n}$ and the object velocity $\boldsymbol{v}_{B}$ is given by

$$
\boldsymbol{D}_{B} \boldsymbol{v}_{B}=\boldsymbol{J}_{C F} \dot{\boldsymbol{\theta}}
$$

where $\boldsymbol{D}_{B} \triangleq \operatorname{col}\left[\boldsymbol{D}_{B 1}, \boldsymbol{D}_{B 2}, \ldots, \boldsymbol{D}_{B n}\right] \in \Re^{3 n \times 6}$ and $\boldsymbol{J}_{C F} \triangleq$ $\operatorname{diag}\left[\boldsymbol{J}_{C F 1}, \boldsymbol{J}_{C F 2}, \ldots, \boldsymbol{J}_{C F n}\right] \in \Re^{3 n \times 3 n}$. Equation (6) represents the kinematic constraint for grasping, and can be rewritten as

$$
\Psi v=0
$$

where

$$
\begin{aligned}
& \boldsymbol{\Psi} \triangleq\left[\begin{array}{ll}
\boldsymbol{D}_{B} & -\boldsymbol{J}_{C F}
\end{array}\right] \in \Re^{3 n \times(3 n+6)} \\
& \boldsymbol{v} \triangleq\left[\begin{array}{ll}
\boldsymbol{v}_{B}^{T} & \dot{\boldsymbol{\theta}}^{T}
\end{array}\right]^{T} \in \Re^{3 n+6} .
\end{aligned}
$$

\section{B. Dynamics of the Hand and Grasped Object}

Dynamic equation of the $i$ th finger is given by

$$
\begin{aligned}
\boldsymbol{\tau}_{i}= & \boldsymbol{M}_{i}\left(\boldsymbol{\theta}_{i}\right) \ddot{\boldsymbol{\theta}}_{i}+\boldsymbol{h}_{i}\left(\boldsymbol{\theta}_{i}, \dot{\boldsymbol{\theta}}_{i}\right) \\
& +\boldsymbol{g}_{i}\left(\boldsymbol{\theta}_{i}\right)+\boldsymbol{J}_{C F i}\left(\boldsymbol{\theta}_{i}\right)^{T} \boldsymbol{f}_{C i}
\end{aligned}
$$

where $\boldsymbol{\tau}_{i} \in \Re^{3}$ and $\boldsymbol{f}_{C i} \in \Re^{3}$ denote the joint driving torque vector and the fingertip force vector of the $i$ th finger, respectively. In (10), $\boldsymbol{M}_{i}\left(\boldsymbol{\theta}_{i}\right) \in \Re^{3 \times 3}$ is the inertia matrix, $\boldsymbol{h}_{i}\left(\boldsymbol{\theta}_{i}, \dot{\boldsymbol{\theta}}_{i}\right) \in \Re^{3}$ means the Coriolis and centrifugal forces, and $\boldsymbol{g}_{i}\left(\boldsymbol{\theta}_{i}\right) \in \Re^{3}$ is the gravity force.

Usually, hand fingers accelerate and decelerate quickly in relatively small workspace and their speed is relatively low. In such cases, the inertia term and the gravity term dominate, and we can neglect the velocity term. Combining (10) for all $n$ fingers, we then get

$$
\boldsymbol{\tau}=\boldsymbol{M}_{F}(\boldsymbol{\theta}) \ddot{\boldsymbol{\theta}}+\boldsymbol{g}_{F}(\boldsymbol{\theta})+\boldsymbol{J}_{C F}(\boldsymbol{\theta})^{T} \boldsymbol{f}_{C}
$$

where $\boldsymbol{\tau} \triangleq \operatorname{col}\left[\boldsymbol{\tau}_{1}, \ldots, \boldsymbol{\tau}_{n}\right] \in \Re^{3 n}, \boldsymbol{M}_{F}(\boldsymbol{\theta}) \triangleq \operatorname{diag}\left[\boldsymbol{M}_{1}, \ldots, \boldsymbol{M}_{n}\right] \in$ $\Re^{3 n \times 3 n}, \boldsymbol{g}_{F}(\boldsymbol{\theta}) \triangleq \operatorname{col}\left[\boldsymbol{g}_{1}, \ldots, \boldsymbol{g}_{n}\right] \in \Re^{3 n}$, and $\boldsymbol{f}_{C} \triangleq \operatorname{col}\left[\boldsymbol{f}_{C 1}\right.$, $\left.\ldots, \boldsymbol{f}_{C n}\right] \in \Re^{3 n}$. Differentiating (6), we get

$$
\boldsymbol{D}_{B} \dot{\boldsymbol{v}}_{B}=\boldsymbol{J}_{C F}(\boldsymbol{\theta}) \ddot{\boldsymbol{\theta}} .
$$

Here also, we neglected the velocity term.

Next, the dynamic equation of the grasped object is given by the following equation:

$$
\boldsymbol{t}_{B}=\boldsymbol{M}_{B} \dot{\boldsymbol{v}}_{B}+\boldsymbol{g}_{B}
$$

where $M_{B} \in \Re^{6 \times 6}$ denotes the inertia tensor of the object and $\boldsymbol{t}_{B} \in$ $\Re^{6}$ is the total force/moment vector applied to the object by the hand. 
For the same reason, we dropped the terms of Coriolis and centrifugal forces in (13).

From the principle of virtual work, we get

$$
\boldsymbol{t}_{B}=\boldsymbol{D}_{B}^{T} \boldsymbol{f}_{C} .
$$

Combining (11), (13), and (14), we get the dynamic equation of the total system as follows:

$$
\boldsymbol{M} \dot{\boldsymbol{v}}+\boldsymbol{g}=\boldsymbol{u}+\boldsymbol{\Psi}^{T} \boldsymbol{f}_{C}
$$

where

$$
\begin{aligned}
\boldsymbol{M} & \triangleq \operatorname{diag}\left[\boldsymbol{M}_{B}, \boldsymbol{M}_{F}\right] \in \Re^{(3 n+6) \times(3 n+6)} \\
\boldsymbol{g} & \triangleq\left[\begin{array}{ll}
\boldsymbol{g}_{B}^{T} & \boldsymbol{g}_{F}^{T}
\end{array}\right]^{T} \in \Re^{3 n+6} \\
\boldsymbol{u} & \triangleq\left[\begin{array}{ll}
\mathbf{0}^{T} & \boldsymbol{\tau}^{T}
\end{array}\right]^{T} \in \Re^{3 n+6}
\end{aligned}
$$

To make the previous equation solvable, we have to combine it with the differentiation of (7) (again, $\dot{\boldsymbol{\Psi}} \boldsymbol{v}$ is dropped), and obtain

$$
\left[\begin{array}{cc}
\boldsymbol{M} & -\boldsymbol{\Psi}^{T} \\
-\boldsymbol{\Psi} & \boldsymbol{O}
\end{array}\right]\left[\begin{array}{c}
\dot{\boldsymbol{v}} \\
\boldsymbol{f}_{C}
\end{array}\right]=\left[\begin{array}{c}
\boldsymbol{u}-\boldsymbol{g} \\
\mathbf{0}
\end{array}\right]
$$

Inverse of the coefficient matrix of (19) is given as

$$
\begin{aligned}
& {\left[\begin{array}{cc}
\boldsymbol{M} & -\boldsymbol{\Psi}^{T} \\
-\boldsymbol{\Psi} & \boldsymbol{O}
\end{array}\right]^{-1}} \\
& =\left[\begin{array}{cc}
\boldsymbol{M}^{-1}-\boldsymbol{M}^{-1} \boldsymbol{\Psi}^{T}\left(\boldsymbol{\Psi} \boldsymbol{M}^{-1} \boldsymbol{\Psi}^{T}\right)^{-1} \boldsymbol{\Psi} \boldsymbol{M}^{-1} & * \\
-\left(\boldsymbol{\Psi} \boldsymbol{M}^{-1} \boldsymbol{\Psi}^{T}\right)^{-1} \boldsymbol{\Psi} \boldsymbol{M}^{-1} & *
\end{array}\right]
\end{aligned}
$$

where we are not interested in $(1,2)$ and $(2,2)$ elements because of the structure of the right-hand side of (19).

Let the following matrix be defined:

$$
\widetilde{\boldsymbol{M}} \triangleq\left(\boldsymbol{D}_{B} \boldsymbol{M}_{B}^{-1} \boldsymbol{D}_{B}^{T}+\boldsymbol{J}_{C F} \boldsymbol{M}_{F}^{-1} \boldsymbol{J}_{C F}^{T}\right)^{-1} .
$$

Then, $(2,1)$ element of $(20)$ is given by the following equation:

$$
\begin{aligned}
-\left(\boldsymbol{\Psi} \boldsymbol{M}^{-1} \boldsymbol{\Psi}^{T}\right)^{-1} \boldsymbol{\Psi} \boldsymbol{M}^{-1} & =\left[\begin{array}{ll}
\widetilde{\boldsymbol{M}} \boldsymbol{D}_{B} \boldsymbol{M}_{B}^{-1} & -\widetilde{\boldsymbol{M}} \boldsymbol{J}_{C F} \boldsymbol{M}_{F}^{-1}
\end{array}\right] \\
& \triangleq\left[\begin{array}{ll}
\boldsymbol{H}_{B} & \boldsymbol{H}_{F}
\end{array}\right] .
\end{aligned}
$$

From (22), we get the general solution of $\boldsymbol{f}_{C}$ for (19) as

$$
\boldsymbol{f}_{C}=\left[\begin{array}{ll}
\boldsymbol{H}_{B} & \boldsymbol{H}_{F}
\end{array}\right]\left[\begin{array}{c}
-\boldsymbol{g}_{B} \\
\boldsymbol{\tau}-\boldsymbol{g}_{F}
\end{array}\right] .
$$

Before computing $(1,1)$ element of $(20)$, we define the following matrix:

$$
\begin{aligned}
\boldsymbol{I} & -\boldsymbol{\Psi}^{T}\left(\boldsymbol{\Psi} \boldsymbol{M}^{-1} \boldsymbol{\Psi}^{T}\right)^{-1} \boldsymbol{\Psi} \boldsymbol{M}^{-1} \\
& =\left[\begin{array}{cc}
\boldsymbol{I}-\boldsymbol{D}_{B}^{T} \widetilde{\boldsymbol{M}} \boldsymbol{D}_{B} \boldsymbol{M}_{B}^{-1} & \boldsymbol{D}_{B}^{T} \widetilde{\boldsymbol{M}} \boldsymbol{J}_{C F} \boldsymbol{M}_{F}^{-1} \\
\boldsymbol{J}_{C F}^{T} \widetilde{\boldsymbol{M}} \boldsymbol{D}_{B} \boldsymbol{M}_{B}^{-1} & \boldsymbol{I}-\boldsymbol{J}_{C F}^{T} \widetilde{\boldsymbol{M}} \boldsymbol{J}_{C F} \boldsymbol{M}_{F}^{-1}
\end{array}\right] \\
& \triangleq\left[\begin{array}{ll}
\boldsymbol{G}_{B B} & \boldsymbol{G}_{B F} \\
\boldsymbol{G}_{F B} & \boldsymbol{G}_{F F}
\end{array}\right] .
\end{aligned}
$$

Using (24), $\boldsymbol{v}$ for (19) can be solved as

$$
\dot{\boldsymbol{v}}=\left[\begin{array}{cc}
\boldsymbol{M}_{B}^{-1} & \mathbf{0} \\
\mathbf{0} & \boldsymbol{M}_{F}^{-1}
\end{array}\right]\left[\begin{array}{ll}
\boldsymbol{G}_{B B} & \boldsymbol{G}_{B F} \\
\boldsymbol{G}_{F B} & \boldsymbol{G}_{F F}
\end{array}\right]\left[\begin{array}{c}
-\boldsymbol{g}_{B} \\
\boldsymbol{\tau}-\boldsymbol{g}_{F}
\end{array}\right] .
$$

Then, the general solution of $\dot{\boldsymbol{v}}_{B}$ is given by

$$
\dot{\boldsymbol{v}}_{B}=\boldsymbol{M}_{B}^{-1}\left[\begin{array}{ll}
\boldsymbol{G}_{B B} & \boldsymbol{G}_{B F}
\end{array}\right]\left[\begin{array}{c}
-\boldsymbol{g}_{B} \\
\boldsymbol{\tau}-\boldsymbol{g}_{F}
\end{array}\right] .
$$

\section{No Gravity Case}

We first formulate the case with no gravity effects, i.e., $\boldsymbol{g}_{B}=\mathbf{0}$ and $\boldsymbol{g}_{F}=\mathbf{0}$. Then, (26) becomes

$$
\dot{\boldsymbol{v}}_{B}=\boldsymbol{M}_{B}^{-1} \boldsymbol{G}_{B F} \boldsymbol{\tau} .
$$

Introducing weighting matrices $\boldsymbol{W}_{\dot{v}}$ and $\boldsymbol{W}_{\tau}$, we normalize both sides of (27) as

$$
\dot{\hat{\boldsymbol{v}}}_{B}=\boldsymbol{W}_{\dot{v}}^{-1} \boldsymbol{M}_{B}^{-1} \boldsymbol{G}_{B F} \boldsymbol{W}_{\tau} \hat{\boldsymbol{\tau}} .
$$

Diagonal matrix $\boldsymbol{W}_{\tau} \in \Re^{3 n \times 3 n}$ consists of the maximum joint torques of each joint and its inverse normalizes the joint torque vector. The normalized (dimensionless) joint torque vector is denoted as $\hat{\tau}$. Similarly, $\boldsymbol{W}_{\dot{v}}$ denotes a weighting matrix for acceleration so that $\dot{\hat{\boldsymbol{v}}}_{B}=\boldsymbol{W}_{\dot{v}}^{-1} \dot{\boldsymbol{v}}_{B}$ becomes a normalized (dimensionless) acceleration vector or a weighted acceleration vector having components with the same physical unit.

From (23), the solution of fingertip force under no gravity becomes

$$
\boldsymbol{f}_{C}=\boldsymbol{H}_{F} \boldsymbol{\tau} .
$$

General solution of $\boldsymbol{f}_{C}$ for (14) can be given by the following equation:

$$
\boldsymbol{f}_{C}=\left(\boldsymbol{D}_{B}^{T}\right)^{+} \boldsymbol{t}_{B}+\boldsymbol{E} \boldsymbol{f}_{I} .
$$

The second term of the right-hand side of (30) represents the internal force component of the fingertip forces. Matrix $\boldsymbol{E} \in \Re^{3 n \times(3 n-6)}$ consists of several unit vectors heading to one contact point from other one. Specifying the magnitude of internal force components by $\boldsymbol{f}_{I}$, fingertip forces in the null space of $\boldsymbol{D}_{B}^{T}$ can be represented in a nonredundant manner. A general method to define $\boldsymbol{E}$ for $n$ fingers is given in [18].

Since $\boldsymbol{E} \boldsymbol{f}_{I}$ is in the null space of $\boldsymbol{D}_{B}^{T}$, it can be represented as

$$
\begin{aligned}
\boldsymbol{E} \boldsymbol{f}_{I} & =\left(\boldsymbol{I}-\left(\boldsymbol{D}_{B}^{T}\right)^{+} \boldsymbol{D}_{B}^{T}\right) \boldsymbol{f}_{C} \\
& =\left(\boldsymbol{I}-\left(\boldsymbol{D}_{B}^{T}\right)^{+} \boldsymbol{D}_{B}^{T}\right) \boldsymbol{H}_{F} \boldsymbol{\tau} .
\end{aligned}
$$

From (31), the internal force component vector is obtained as

$$
\boldsymbol{f}_{I}=\boldsymbol{E}^{+}\left(\boldsymbol{I}-\left(\boldsymbol{D}_{B}^{T}\right)^{+} \boldsymbol{D}_{B}^{T}\right) \boldsymbol{H}_{F} \boldsymbol{W}_{\tau} \hat{\boldsymbol{\tau}}
$$

From (28) and (32), we finally get the following equation:

$$
\begin{aligned}
{\left[\begin{array}{c}
\dot{\boldsymbol{v}}_{B} \\
\boldsymbol{f}_{I}
\end{array}\right] } & =\left[\begin{array}{c}
\boldsymbol{W}_{\dot{v}}^{-1} \boldsymbol{M}_{B}^{-1} \boldsymbol{G}_{B F} \boldsymbol{W}_{\tau} \\
\boldsymbol{E}^{+}\left(\boldsymbol{I}-\left(\boldsymbol{D}_{B}^{T}\right)^{+} \boldsymbol{D}_{B}^{T}\right) \boldsymbol{H}_{F} \boldsymbol{W}_{\tau}
\end{array}\right] \hat{\boldsymbol{\tau}} \\
& \triangleq\left[\begin{array}{c}
\boldsymbol{\Gamma}_{B} \\
\boldsymbol{\Gamma}_{F}
\end{array}\right] \hat{\boldsymbol{\tau}} .
\end{aligned}
$$

This is the basic equation to derive the dynamic manipulability of multifingered grasping in Section III.

\section{Effects of Gravity}

In the previous section, we neglected the gravity effects. Here, we calculate the offsets of object accelerations and the internal forces due to gravity.

Let us consider the case where only gravity forces are applied. First, from (26), the acceleration of the grasped object is given as

$$
\dot{\boldsymbol{v}}_{B g}=\boldsymbol{M}_{B}^{-1}\left[\begin{array}{ll}
\boldsymbol{G}_{B B} & \boldsymbol{G}_{B F}
\end{array}\right]\left[\begin{array}{l}
-\boldsymbol{g}_{B} \\
-\boldsymbol{g}_{F}
\end{array}\right] .
$$

This is the offset of the grasped object acceleration due to gravity. 


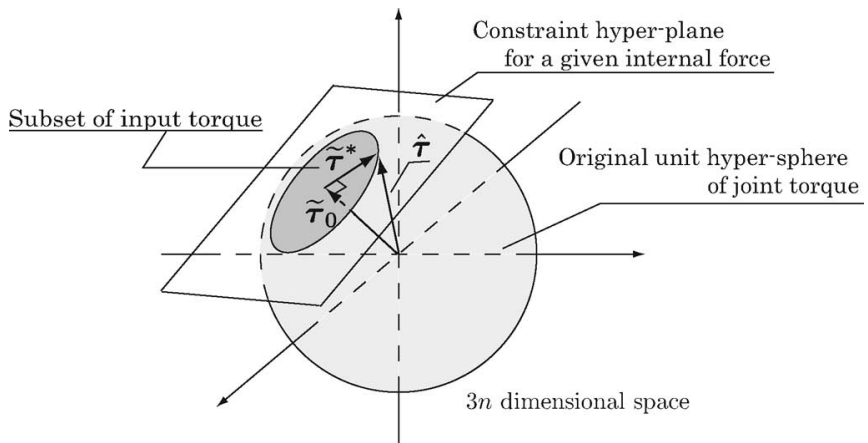

Fig. 2. Subset of input torque under the constraint of a given constant internal force.

Similarly, from (23), the fingertip force under gravity is given as

$$
\boldsymbol{f}_{C g}=\left[\begin{array}{ll}
\boldsymbol{H}_{B} & \boldsymbol{H}_{F}
\end{array}\right]\left[\begin{array}{l}
-\boldsymbol{g}_{B} \\
-\boldsymbol{g}_{F}
\end{array}\right] .
$$

From (31), the offset value of internal force components due to gravity is given as

$$
\boldsymbol{f}_{I g}=\boldsymbol{E}^{+}\left(\boldsymbol{I}-\left(\boldsymbol{D}_{B}^{T}\right)^{+} \boldsymbol{D}_{B}^{T}\right) \boldsymbol{f}_{C g} .
$$

Positive components of $\boldsymbol{f}_{I g}$ mean that the gravity effects contribute to increasing the internal force along these components, and vice versa for negative components.

\section{DYNAMIC MANIPULABILITY OF MULTIFINGERED GRASPING}

\section{A. Definition of Dynamic Manipulability of Multifingered Grasping}

Considering the relation among $\hat{\boldsymbol{\tau}}, \dot{\hat{\boldsymbol{v}}}_{B}$, and $\boldsymbol{f}_{I}$ in (33), the joint torque that realizes a given object acceleration cannot be determined uniquely, because of the existence of the internal force component. However, keeping the internal force vector $\boldsymbol{f}_{I}$ constant, we can obtain a one-to-one relation between object accelerations and joint torques. It would be possible to define the dynamic manipulability of multifingered grasping as a set of possible maximum accelerations of the grasped object with all possible fingertip forces that satisfy the friction condition, where internal force may change depending on the direction in which the grasped object is accelerated. Unfortunately, such a set cannot be obtained analytically. In this paper, we define the dynamic manipulability of multifingered grasping as a function of internal forces so that the manipulability ellipsoid is analytically defined like the original manipulability measure.

Note that we must set the internal forces so that all possible fingertip forces satisfy the friction condition between the fingertip and the object to make the grasping feasible. In the following formulation, we assume that a given internal force satisfies the friction condition and the fingertips keep contact without any slippage. In Section III-B, we will discuss how to find such internal forces.

With a fixed internal force vector $\boldsymbol{f}_{I}$, the equation $\boldsymbol{\Gamma}_{F} \hat{\boldsymbol{\tau}}=\boldsymbol{f}_{I}$, which is obtained from (33), represents $(3 n-6)$-dimensional constraint. Under such constraint, joint torque vectors in $3 n$-dimensional space are constraint on the 6-D hyperplane, as shown in Fig. 2, where the unit hypersphere of $\hat{\tau}$ is cut off by this hyperplane. Namely, by keeping the internal force component constant, $\hat{\tau}$ is constrained within the intercept region shown in Fig. 2, thus resulting in a 6-D hypersphere in $3 n$-dimensional space. The resultant object acceleration set by the set of joint torques inside this 6-D hypersphere becomes a 6-D ellipsoid in $3 n$-dimensional space, as shown in Fig. 3, which has an offset in the direction of $\boldsymbol{f}_{I}$.

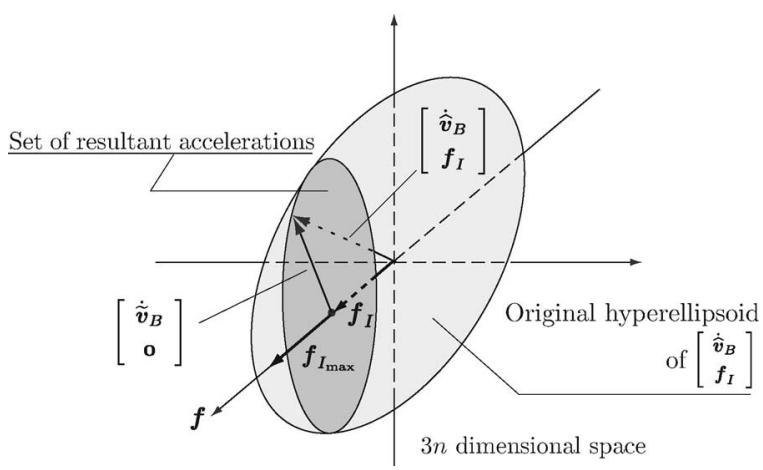

Fig. 3. Set of resultant accelerations under the constraint of a given constant internal force.

Now, let $\widetilde{\boldsymbol{\tau}}_{0}$ be a vector from the center of the original unit hypersphere to the center of the intercept region, and let $\widetilde{\tau}^{*}$ be a vector perpendicular to $\widetilde{\tau}_{0}$, as shown in Fig. 2 . Then any joint torque that results in the same given internal force can be expressed as $\hat{\tau}=\widetilde{\tau}_{0}+\widetilde{\boldsymbol{\tau}}^{*}$.

The general solution of $\boldsymbol{f}_{I}=\boldsymbol{\Gamma}_{F} \hat{\boldsymbol{\tau}}$ is given by $\hat{\boldsymbol{\tau}}=\boldsymbol{\Gamma}_{F}^{+} \boldsymbol{f}_{I}+(\boldsymbol{I}-$ $\left.\boldsymbol{\Gamma}_{F}^{+} \boldsymbol{\Gamma}_{F}\right) \boldsymbol{\xi}$, where $\boldsymbol{\Gamma}_{F}^{+}$denotes the pseudoinverse of $\boldsymbol{\Gamma}_{F}$. Since the first term gives the minimum-norm solution to realize the given $\boldsymbol{f}_{I}$, we get $\widetilde{\boldsymbol{\tau}}_{0}=\boldsymbol{\Gamma}_{F}^{+} \boldsymbol{f}_{I}$ and $\widetilde{\boldsymbol{\tau}}^{*}=\left(\boldsymbol{I}-\boldsymbol{\Gamma}_{F}^{+} \boldsymbol{\Gamma}_{F}\right) \boldsymbol{\xi}$, where $\boldsymbol{\xi} \in \Re^{3 n}$ is an arbitrary vector. Then, (33) can be rewritten as

$$
\begin{gathered}
{\left[\begin{array}{c}
\dot{\boldsymbol{v}}_{B} \\
\boldsymbol{f}_{I}
\end{array}\right]=\left[\begin{array}{c}
\boldsymbol{\Gamma}_{B} \\
\boldsymbol{\Gamma}_{F}
\end{array}\right] \hat{\boldsymbol{\tau}}=\left[\begin{array}{c}
\boldsymbol{\Gamma}_{B} \\
\boldsymbol{\Gamma}_{F}
\end{array}\right]\left(\widetilde{\boldsymbol{\tau}}_{0}+\widetilde{\boldsymbol{\tau}}^{*}\right)} \\
=\left[\begin{array}{c}
\boldsymbol{\Gamma}_{B} \boldsymbol{\Gamma}_{F}^{+} \boldsymbol{f}_{I} \\
\boldsymbol{f}_{I}
\end{array}\right]+\left[\begin{array}{c}
\boldsymbol{\Gamma}_{B}\left(\boldsymbol{I}-\boldsymbol{\Gamma}_{F}^{+} \boldsymbol{\Gamma}_{F}\right) \boldsymbol{\xi} \\
\mathbf{0}
\end{array}\right] .
\end{gathered}
$$

The necessary and sufficient condition to obtain (37) is that matrix $\boldsymbol{\Gamma}_{F}^{+}$ is full-rank.

Since $\widetilde{\boldsymbol{\tau}}_{0}$ and $\widetilde{\boldsymbol{\tau}}^{*}$ are perpendicular, we get

$$
\left\|\widetilde{\boldsymbol{\tau}}^{*}\right\|^{2}=\|\hat{\boldsymbol{\tau}}\|^{2}-\left\|\tilde{\boldsymbol{\tau}}_{0}\right\|^{2} \leq 1-\left\|\boldsymbol{\Gamma}_{F}^{+} \boldsymbol{f}_{I}\right\|^{2}
$$

From (38), we set an arbitrary vector $\boldsymbol{\xi}$ as follows:

$$
\boldsymbol{\xi}=\left(1-\left\|\boldsymbol{\Gamma}_{F}^{+} \boldsymbol{f}_{I}\right\|^{2}\right)^{1 / 2} \hat{\boldsymbol{\tau}}^{*}
$$

where $\hat{\tau}^{*}$ denotes an arbitrary vector within the $3 n$-dimensional unit hypersphere. Since $\widetilde{\boldsymbol{\tau}}^{*}=\left(\boldsymbol{I}-\boldsymbol{\Gamma}_{F}^{+} \boldsymbol{\Gamma}_{F}\right) \boldsymbol{\xi}$, we get $\left\|\tilde{\boldsymbol{\tau}}^{*}\right\| \leq\|\boldsymbol{\xi}\|$. Therefore, $\widetilde{\boldsymbol{\tau}}^{*}$ with $\boldsymbol{\xi}$ given by (39) always satisfies the norm condition of (38).

Substituting (39) into (37), we get

$$
\dot{\widetilde{\boldsymbol{v}}}_{B}=\left(1-\left\|\boldsymbol{\Gamma}_{F}^{+} \boldsymbol{f}_{I}\right\|^{2}\right)^{1 / 2} \boldsymbol{\Gamma}_{B}\left(\boldsymbol{I}-\boldsymbol{\Gamma}_{F}^{+} \boldsymbol{\Gamma}_{F}\right) \hat{\boldsymbol{\tau}}^{*}
$$

where $\dot{\widetilde{\boldsymbol{v}}}_{B}=\dot{\hat{\boldsymbol{v}}}_{B}-\boldsymbol{\Gamma}_{B} \boldsymbol{\Gamma}_{F}^{+} \boldsymbol{f}_{I}$ denotes the offset acceleration. Although $\hat{\tau}^{*}$ spans within the $3 n$-dimensional unit hypersphere, it is mapped to the null space of $\boldsymbol{\Gamma}_{F}$ by $\left(\boldsymbol{I}-\boldsymbol{\Gamma}_{F}^{+} \boldsymbol{\Gamma}_{F}\right)$, thus resulting in a 6-D unit hypersphere in the intercept region of $\widetilde{\boldsymbol{\tau}}^{*}$.

From (40), we define the dynamic manipulability matrix of multifingered grasping $\boldsymbol{\Gamma}_{G}$, which specifies the mapping from a unit sphere to an ellipsoid of the grasped object acceleration, as follows:

$$
\boldsymbol{\Gamma}_{G}=\left(1-\left\|\boldsymbol{\Gamma}_{F}^{+} \boldsymbol{f}_{I}\right\|^{2}\right)^{1 / 2} \boldsymbol{\Gamma}_{B}\left(\boldsymbol{I}-\boldsymbol{\Gamma}_{F}^{+} \boldsymbol{\Gamma}_{F}\right) .
$$


All sets of the realizable offset accelerations of the grasped object $\dot{\widetilde{\boldsymbol{v}}}_{B}$ by all sets of the normalized inputs $\hat{\boldsymbol{\tau}}^{*}\left(\left\|\hat{\boldsymbol{\tau}}^{*}\right\| \leq 1\right)$ form an ellipsoid, which is given as

$$
\dot{\widetilde{\boldsymbol{v}}}_{B}^{T}\left(\boldsymbol{\Gamma}_{G}^{+}\right)^{T} \boldsymbol{\Gamma}_{G}^{+} \dot{\widetilde{\boldsymbol{v}}}_{B} \leq 1 \quad \text { and } \quad \dot{\widetilde{\boldsymbol{v}}}_{B} \in \Re\left(\boldsymbol{\Gamma}_{G}\right)
$$

where $\Re\left(\boldsymbol{\Gamma}_{G}\right)$ denotes the range of $\boldsymbol{\Gamma}_{G}$. This ellipsoid is termed a "dynamic manipulability ellipsoid of multifingered grasping."

Similarly to the original dynamic manipulability, dynamic manipulability measure of multifingered grasping is defined as

$$
w_{G}=\sqrt{\operatorname{det}\left(\boldsymbol{\Gamma}_{G} \boldsymbol{\Gamma}_{G}^{T}\right)} .
$$

\section{B. Internal Forces}

Since the dynamic manipulability of multifingered grasping was defined as a function of the internal force $f_{I}$, as shown in (41), we need to specify the internal force $f_{I}$. The internal force should be determined so that all possible fingertip forces under a given internal force $\boldsymbol{f}_{I}$ satisfy the friction condition. From (29), such a set of $\boldsymbol{f}_{C}$ with a given $f_{I}$ is given as

$$
\boldsymbol{f}_{C}=\boldsymbol{H}_{F} \boldsymbol{W}_{\tau}\left(\boldsymbol{\Gamma}_{F}^{+} \boldsymbol{f}_{I}+\left(1-\left\|\boldsymbol{\Gamma}_{F}^{+} \boldsymbol{f}_{I}\right\|^{2}\right)^{1 / 2}\left(\boldsymbol{I}-\boldsymbol{\Gamma}_{F}^{+} \boldsymbol{\Gamma}_{F}\right) \hat{\boldsymbol{\tau}}^{*}\right) .
$$

Note that (44) gives a set of $\boldsymbol{f}_{C}$ under no gravity effect, and we have to add $\boldsymbol{f}_{C g}$ given by (35) to (44) when we need to consider the gravity effect.

Considering that (44) gives an ellipsoid with an offset in the fingertip force space, we can check the friction condition by scanning all possible fingertip forces on this ellipsoid ( $n$-time 2-D searching), which is faster than scanning all possible joint torques in the joint space [ $(3 n-1)$ dimensional search]. If we approximate the friction cone by a convex polyhedral cone, we can check the contact condition analytically by checking the collision between the ellipsoid and each plane consisting of the convex polyhedral cone.

When evaluating $w_{G}$ defined by (43), one can apply any internal forces as long as all possible fingertip forces satisfy the friction condition. Clearly, as $\left\|\boldsymbol{f}_{I}\right\|$ becomes larger, the volume of the ellipsoid becomes smaller. To avoid conservative evaluations, usually the internal force is chosen so that it maximizes, for example, $w_{G}$ under the friction condition constraint. ${ }^{2}$ Although it would be possible to search for an optimal $\boldsymbol{f}_{I}$ in $(3 n-6)$-dimensional space, we can specify a feasible internal force basis that satisfies the friction condition, as shown in [22], to restrict the search space. From (38), $\boldsymbol{f}_{I}$ must satisfy the following inequality:

$$
\left\|\boldsymbol{\Gamma}_{F}^{+} \boldsymbol{f}_{I}\right\|^{2} \leq 1 \quad \text { or } \quad \boldsymbol{f}_{I}^{T} \boldsymbol{\Gamma}_{F}^{+T} \boldsymbol{\Gamma}_{F}^{+} \boldsymbol{f}_{I} \leq 1 .
$$

Inequality (45) means that the internal forces have upper limitations and form an ellipsoid in the internal force space. Let $\boldsymbol{f}_{I_{\max }}$ be the maximum internal force along with the selected internal force basis satisfying (45), we can represent $\boldsymbol{f}_{I}$ as follows:

$$
\boldsymbol{f}_{I}=\hat{f}_{I} \boldsymbol{f}_{I_{\mathrm{max}}}, \quad 0 \leq \hat{f}_{I} \leq 1
$$

where $\hat{f}_{I}$ is a scalar value representing the normalized magnitude of the internal force along with the selected basis. Then, the problem is reduced to find a minimum $\hat{f}_{I}$ under the friction condition constraint. Note, however, that the minimum $\hat{f}_{I}$ gives a suboptimal internal force because the optimal one may not exist along with the selected basis.

\footnotetext{
${ }^{2}$ One should keep in mind that such an optimal case is critical in a sense that some of the fingertip forces may lie on the boundary of the friction cone.
}

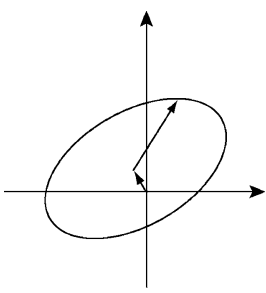

(a)

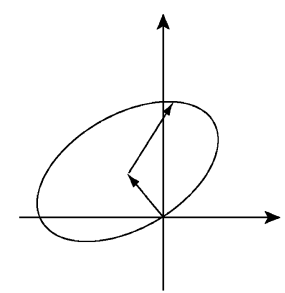

(b)

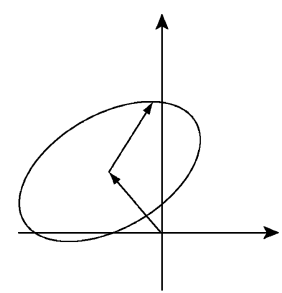

(c)
Fig. 4. Offset and omnidirectionality of ellipsoid. (a) Omnidirectional. (b) Critical. (c) Nonomnidirectional.

\section{Offset and Omnidirectional Property of the Manipulability Ellipsoids}

Chiacchio et al. showed that the gravity force simply offsets the dynamic manipulability property [5], [6]. In our previous study [12], we neglected the gravity effect and did not consider the effect of offsets of the dynamic manipulability property due to gravity and internal forces. Here, we explicitly discuss on this point.

The offset of the dynamic manipulability property is defined by the following equation:

$$
\dot{\hat{\boldsymbol{v}}}_{B}=\boldsymbol{\Gamma}_{G} \hat{\boldsymbol{\tau}}^{*}+\boldsymbol{b}
$$

where $\boldsymbol{b}$ denotes the offset. Equation (47) means that the resultant (normalized or weighted) acceleration can be represented by an ellipsoid defined by (42) and the offset $\boldsymbol{b}$.

Total offset is given as

$$
\boldsymbol{b}=\boldsymbol{b}_{g}+\boldsymbol{b}_{I}
$$

where $\boldsymbol{b}_{g}$ denotes the offset by the gravity and $\boldsymbol{b}_{I}$ is the offset by the internal forces. From (34), $\boldsymbol{b}_{g}$ is given as

$$
\boldsymbol{b}_{g}=-\boldsymbol{W}_{\dot{v}}^{-1} \dot{\boldsymbol{v}}_{B g} .
$$

From (37), $\boldsymbol{b}_{I}$ is given as

$$
\boldsymbol{b}_{I}=\boldsymbol{\Gamma}_{B} \boldsymbol{\Gamma}_{F}^{+} \boldsymbol{f}_{I} .
$$

Note that $\boldsymbol{b}_{I}$ depends on how much internal force we specify, but $\boldsymbol{b}_{g}$ does not. Also note that under gravity, we have an offset of internal forces $\boldsymbol{f}_{I g}$ given by (36). Let $\boldsymbol{f}_{I_{d}}$ be the desired value of the internal forces, internal forces that should be realized by joint torque is given as

$$
\boldsymbol{f}_{I}=\boldsymbol{f}_{I_{d}}-\boldsymbol{f}_{I g}
$$

From (42), dynamic manipulability ellipsoid with the offset is given as

$$
\left(\dot{\hat{\boldsymbol{v}}}_{B}-\boldsymbol{b}\right)^{T}\left(\boldsymbol{\Gamma}_{G}^{+}\right)^{T} \boldsymbol{\Gamma}_{G}^{+}\left(\dot{\hat{\boldsymbol{v}}}_{B}-\boldsymbol{b}\right) \leq 1 .
$$

The ability to accelerate in all directions, namely omnidirectionality, depends on the offset, and it can be checked as follows:

$$
\eta \triangleq \boldsymbol{b}^{T}\left(\boldsymbol{\Gamma}_{G}^{+}\right)^{T} \boldsymbol{\Gamma}_{G}^{+} \boldsymbol{b} \begin{cases}<1, & \text { omnidirectional } \\ =1, & \text { critical } \\ >1, & \text { nonomnidirectional. }\end{cases}
$$

Fig. 4 illustrates three cases given by (53). Then, we introduce a new manipulability measure with a penalty as

$$
\bar{w}_{G}=\xi w_{G}
$$

where the penalty factor $\xi$ is defined as

$$
\xi \triangleq \begin{cases}1-\eta, & \text { if } \eta \leq 1 \\ 0, & \text { otherwise. }\end{cases}
$$




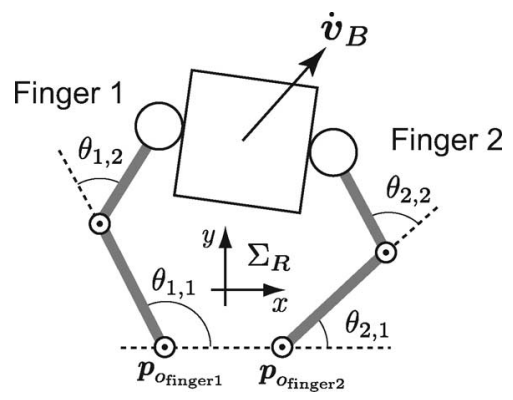

Fig. 5. Two-fingered hand.

\section{Discussions}

Here, we discuss two issues related to manipulability measure. Note that these issues are not specific to our measure, but would be related to some existing manipulability measures as well.

1) Dependency on Units and the Reference Point: Doty et al. [11] argued that the conventional manipulability measure has several problems, such as the dependency of the choice of units and the dependency of the chosen reference frame.

The proposed measure in this paper is consistent with the choice of unit because we introduced weighting matrices $\boldsymbol{W}_{\tau}$ and $\boldsymbol{W}_{\dot{v}}$. To make the object acceleration dimensionless, $\boldsymbol{W}_{\dot{v}}$ can be a diagonal matrix consisting of the maximum accelerations for each component. We can also set it as $\boldsymbol{W}_{\dot{v}}={\sqrt{\boldsymbol{M}_{B}}}^{-1}$ so that the weighted acceleration $\dot{\hat{\boldsymbol{v}}}_{B}=\boldsymbol{W}_{\dot{v}}^{-1} \dot{\boldsymbol{v}}_{B}$ has components with the same physical unit.

When we change the reference point, we have to change $\boldsymbol{W}_{\dot{v}}$ accordingly so that the manipulability measure is invariant. Note that the formulation in this paper corresponds to the weighted generalized inverse suggested in [10] and [11] as long as the hand system is nonsingular.

2) Polytope Versus Ellipsoid: The manipulability polytope is an alternative way of evaluating the maximum achievable task variable (in our case, acceleration) under the constraint of joint variable (in our case, joint torque) where each element is independently bounded [7].

Although the manipulability ellipsoid can be obtained analytically and handled more easily than polytope, one should note that the manipulability ellipsoid becomes an approximation of the polytope when each element of the joint variables is bounded independently. Actually, Melchiorri pointed out that the optimal direction obtained from the manipulability ellipsoid (i.e., the largest principal axis of the ellipsoid) differs from the optimal direction given by the polytope [15].

\section{NUMERICAL EXAMPLE}

In this section, we show some numerical examples using the proposed measure. Although the formulations in the previous sections can handle a general $n$-fingered hand in 3-D space, we decided to use a two-fingered robot hand in 2-D space to demonstrate the effectiveness of the proposed measure simply and clearly.

\section{A. Parameter Setting}

Fig. 5 illustrates the robot hand used in the following examples. This robot hand has two fingers, each of which has two joints and the grasped object can make planer motions only. Therefore, the formulation in the previous sections was converted to the planer motion case with two fingers, where $\dot{\hat{\boldsymbol{v}}}_{B} \in \Re^{3}$ and $\boldsymbol{f}_{I}$ becomes a scalar $f_{I}$.

The location of the first joint of the left finger (finger 1) with respect to the reference coordinate frame shown in Fig. 5 is $\boldsymbol{p}_{o_{\text {finger } 1}}=$
TABLE I

PARAMETERS OF THE HAND MODEL

\begin{tabular}{|l||c|c|}
\hline \multicolumn{1}{|c||}{} & \multicolumn{2}{c|}{ Finger 1 \& Finger 2 } \\
\cline { 2 - 3 } & Link1 & Link2 \\
\hline Link length $[\mathrm{m}]$ & 0.06 & 0.05 \\
Mass $\left[10^{-3} \mathrm{~kg}\right]$ & 54.5 & 45.4 \\
Moment of inertia $\left[10^{-6} \mathrm{kgm}^{2}\right]$ & 67.7 & 39.7 \\
\hline Maximum motor torque $[\mathrm{Nm}]$ & \multicolumn{2}{c|}{0.0349} \\
Rotor inertia $\left[10^{-7} \mathrm{kgm}^{2}\right]$ & \multicolumn{2}{|c|}{1.15} \\
Reduction gear ratio & \multicolumn{2}{c|}{6.4} \\
\hline
\end{tabular}

$[-0.025,-0.025]^{T} \mathrm{~m}$. The location of the first joint of the right finger (finger 2) is $\boldsymbol{p}_{o_{\text {finger } 2}}=[0.025,-0.025]^{T} \mathrm{~m}$. A circular pad with $0.011 \mathrm{~m}$ radius is attached to each fingertip. Parameters of the hand model are summarized in Table I.

Joint angles for each finger are defined, as shown in Fig. 5. The motion range for each joint is set as $20^{\circ} \leq \theta_{1,1} \leq 180^{\circ},-120^{\circ} \leq$ $\theta_{1,2} \leq 0^{\circ}, 0^{\circ} \leq \theta_{2,1} \leq 160^{\circ}$, and $0^{\circ} \leq \theta_{2,2} \leq 120^{\circ}$. As can be seen from the motion range of the second joints, we assume that finger 1 is always elbow-up while finger 2 is always elbow-down. We also assume that actuators (motors) are located at the corresponding joint and drive the joint through a reduction gear with the ratio shown in Table I.

The grasped object is a $0.057-\mathrm{m}$ square box, which has $0.02 \mathrm{~kg}$ mass with uniform material (therefore, moment of inertia is $1.083 \times$ $10^{-5} \mathrm{~kg} \cdot \mathrm{m}^{2}$ ). The friction coefficient between the object and the fingertip was set to $\mu=0.8$. The reference point (the origin of $\Sigma_{B}$ ) is located at the gravity center of the object.

The weighting matrix for object accelerations was set to $\boldsymbol{W}_{\dot{v}}={\sqrt{\boldsymbol{M}_{B}}}^{-1}$, as explained in Section III-D. Using the values of the grasped object inertia, we actually $\quad$ set $\quad \boldsymbol{W}_{\dot{v}}=\operatorname{diag}\left[1 / \sqrt{0.02}\left(\right.\right.$ in $\left._{\mathrm{kg}}^{-1 / 2}\right), 1 / \sqrt{0.02}\left(\mathrm{in} \mathrm{kg}^{-1 / 2}\right)$, $\left.1 / \sqrt{1.083 \times 10^{-5}}\left(\mathrm{in}_{\mathrm{kg}}^{-1 / 2} \cdot \mathrm{m}^{-1}\right)\right]$. The weighting matrix for joint torque $\boldsymbol{W}_{\tau}$ was set with the values shown in Table I considering the reduction ratio.

To evaluate the manipulability at each point inside the working space, we assume that the top and bottom sides of the object always face toward the origin of the reference frame, and the fingers always contact with the object at the middle point of the side face.

\section{B. Manipulability Ellipsoids and Measures}

Fig. 6 illustrates the dynamic manipulability ellipsoids of multifingered grasping when the hand grasps the object located at $[x, y]^{T}=$ $[-0.02,0.07]^{T} \mathrm{~m}$ with different internal forces, i.e., $f_{I}=\hat{f}_{I} f_{I_{\max }}$ with $\hat{f}_{I}=0,0.2,0.4,0.6,0.8$, and 0.95 , under no gravity effect. Note that the maximum internal force that satisfies (45) at this point is $f_{I_{\max }}=0.362 \mathrm{~N}$. Also note that ellipsoids in Fig. 6 show only translation components, i.e., they are the projections of 3-D ellipsoids onto the 2-D linear acceleration space.

Fig. 7 illustrates fingertip force ellipsoids given by (44) with different internal forces $\hat{f}_{I}=0.4,0.6,0.8$, and 0.51 at the finger configuration corresponding to that in Fig. 6. We found that when $\hat{f}_{I}>0.509$, the internal force becomes feasible, i.e., all possible fingertip forces satisfy the grasp condition with $\mu=0.8$.

In Fig. 6, we deliberately show ellipsoids with different internal forces, including unfeasible ones and the ones larger than the optimal one (in this case, $\hat{f}_{I}=0.51$ ), which usually means waste of joint torques. Then, the figure clearly shows that the size of ellipsoid becomes smaller and the amount of offset becomes larger as the larger internal force is applied. When $\hat{f}_{I}$ is very close to 1.0 (namely $f_{I}$ is very close to $f_{I_{\mathrm{m} \mathrm{ax}}}$ ), the size of ellipsoid becomes very small, meaning that the hand no longer has much extra joint torque to adjust the acceleration of the 


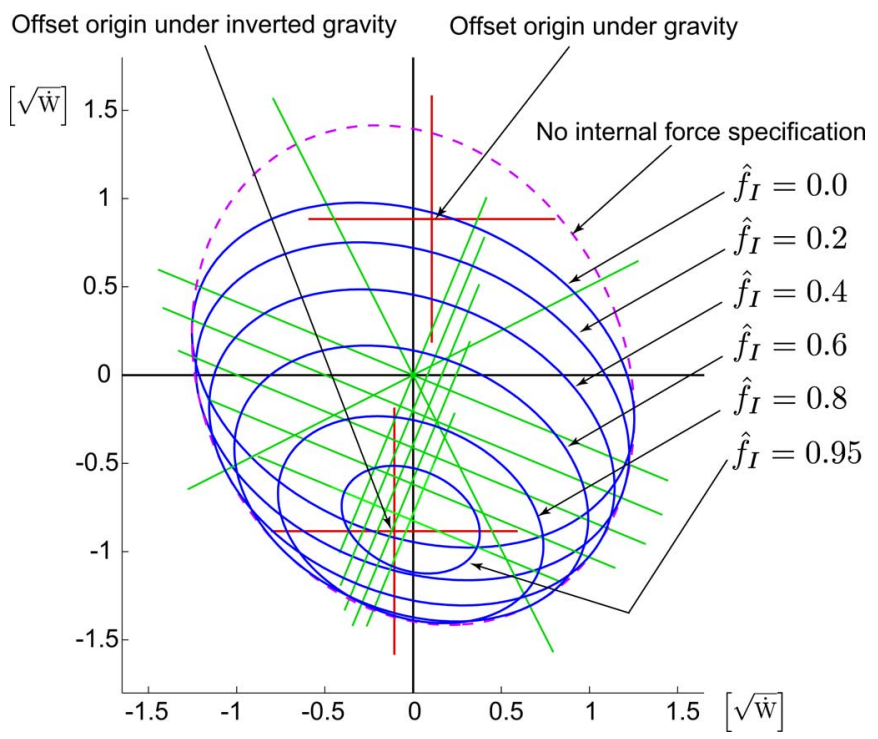

Fig. 6. Dynamic manipulability ellipsoids of multifingered grasping and the effect of internal force and gravity on them.

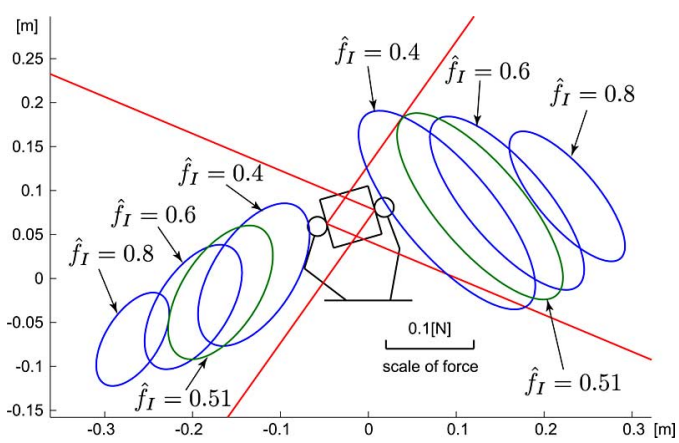

Fig. 7. Fingertip force ellipsoids with different internal forces.

grasped object. Fig. 6 also illustrates the manipulability ellipsoid where no internal force is considered. Note that this ellipsoid is larger than the ellipsoid with $\hat{f}_{I}=0$ since it can include the case where the internal force component becomes negative. Of course, all of the ellipsoids with various internal forces are inside this ellipsoid.

In Fig. 6, the origins of $\dot{\hat{\boldsymbol{v}}}_{B}$ offset under gravity and inverted gravity, which corresponds to $-\boldsymbol{b}_{g}$, where $\boldsymbol{b}_{g}$ was defined by (49), are also drawn due to the space limitation. When gravity is applied downward, the origin is shifted upward and most of the ellipsoids become nonomnidirectional. When the gravity is applied upward (inverted gravity), on the other hand, the origin is shifted downward and most of the ellipsoids become omnidirectional because the offset due to the gravity cancels the offset due to the internal force.

Fig. 8 shows contour maps of the manipulability measure considering omnidirectionality $\bar{w}_{G}$, which is defined by (54) in two different gravity conditions. We calculated $\bar{w}_{G}$ in a fan-shaped area with radius $R=0.065 \mathrm{~m}$ and central angle $120^{\circ}$, which approximates the working volume of the hand system.

Fig. 8(a) is the map of $\bar{w}_{G}$ under no gravity. Fig. 8(b) illustrates the case when gravity is applied upward. When drawing these maps, we used the minimum feasible (namely, optimal) internal forces at each location. We do not show the map under the gravity downward because every point in the working volume becomes nonomnidirectional in this gravity condition.

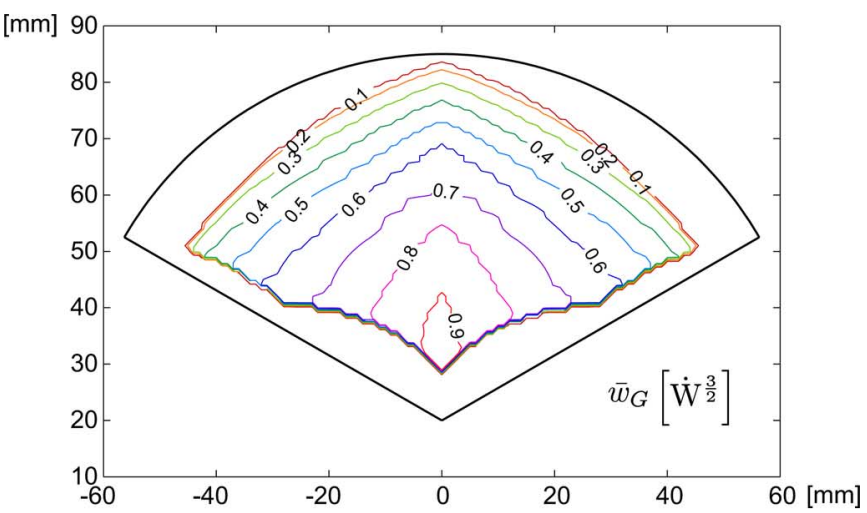

(a)

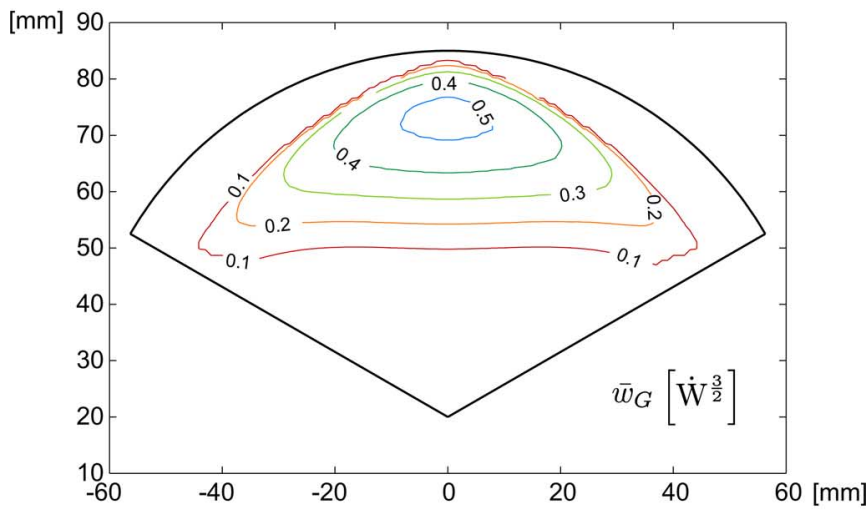

(b)

Fig. 8. Contour map of manipulability measure considering omnidirectionality. (a) No gravity. (b) Under inverted gravity.

Unlike the case of gravity downward, some points in the working volume become omnidirectional under the gravity upward, because the offsets due to the internal force and gravity cancel each other, as we have seen in Fig. 6. Note that the contour maps of $w_{G}$, which simply evaluates the volume of the ellipsoid at each point, are almost the same among these three cases, including the downward gravity case. This means that the offsets due to the gravity and the internal force drastically change the manipulability measure $\bar{w}_{G}$.

\section{CONCLUSION}

In this paper, we extended the concept of the original dynamic manipulability to evaluate the dynamic property of multifingered grasping systems consisting of a multifingered hand and a grasped object, and proposed a measure of dynamic manipulability of multifingered grasping. It was shown that applying all possible joint torques under a constant internal force constraint, the set of resultant accelerations of the grasped object forms an ellipsoid (dynamic manipulability ellipsoid of multifingered grasping). Similar to the original dynamic manipulability measure, we proposed the dynamic manipulability measure of multifingered grasping as the volume of this ellipsoid. To evaluate the dynamic manipulability of a multifingered system, one has to specify certain internal forces under which all possible fingertip forces satisfy the friction condition.

With the proposed measure, it was clearly shown that the internal forces not only affect the volume of the ellipsoid, but also the amount of offset of the ellipsoid, while the gravity forces simply induce an offset. A new measure, i.e., omnidirectionality, was also introduced to add a 
penalty to the original manipulability measure, which simply evaluates the volume of the ellipsoid, depending on how much the ellipsoid is offset.

Numerical examples by using a simple two-fingered robot hand were shown to demonstrate the effectiveness of the proposed measure, including the effect of offsets of the ellipsoids.

Like the original dynamic manipulability measure, the proposed measure is a local index. It would be possible to define a global index by integrating the local index over the workspace of the system considering how frequently the robot visits each point [16].

\section{ACKNOWLEDGMENT}

The authors acknowledge some valuable comments from the anonymous reviewers from which they conceived the idea of omnidirectionality. This research was supported by Grants-in-Aid for Scientific Research (Scientific Research (B) No. 19300068) sponsored by Japan Society for the Promotion of Science (JSPS).

\section{REFERENCES}

[1] A. Bicchi, C. Melchiorri, and D. Balluchi, "On the mobility and manipulability of general multiple limb robots," IEEE Trans. Robot. Autom. vol. 11, no. 2, pp. 253-260, Apr. 1995.

[2] A. Bicchi, D. Prattichizzo, and C. Melchiorri, "Force and dynamic manipulability for cooperating robot systems," in Proc. IEEE/RSJ Int. Conf. Intell. Robots Syst. (IROS), 1997, vol. 3, pp. 1479-1484.

[3] A. Bicchi and D. Prattichizzo, "Manipulability of cooperating robots with unactuated joints and closed-chain mechanisms," IEEE Trans. Robot. Autom., vol. 16, no. 4, pp. 336-345, Aug. 2000.

[4] P. Chiacchio, S. Chiaverini, L. Sciavicco, and B. Siciliano, "Global task space manipulability ellipsoids for multiple-arm systems," IEEE Trans. Robot. Autom., vol. 7, no. 5, pp. 678-685, Oct. 1991

[5] P. Chiacchio, S. Chiaverini, L. Sciavicco, and B. Siciliano "Task space dynamic analysis of multiarm system configurations," Int. J. Robot. Res., vol. 10, no. 6, pp. 708-715, 1991.

[6] P. Chiacchio, S. Chiaverini, L. Sciavicco, and B. Siciliano, "Influence of gravity on the manipulability ellipsoid for robot arms," ASME J. Dyn. Syst., Meas., Control, vol. 114, pp. 723-727, 1992.

[7] P. Chiacchio, Y. Bouffard-Vercelli, and F. Pierrot, "Force polytope and force ellipsoid for redundant manipulators," J. Robot. Syst., vol. 14, no. 8, pp. 613-620, 1997.

[8] P. Chiacchio, "A new dynamic manipulability ellipsoid for redundant manipulators," Robotica, vol. 18, pp. 381-387, 2000.

[9] A. B. A. Cole, J. E. Hauser, and S. S. Satry, "Kinematics and control of multifingered hands with rolling contact," IEEE Trans. Autom. Control, vol. 34, no. 4, pp. 398-404, Apr. 1989.

[10] K. L. Doty, C. Melchiorri, and C. Bonivento, "A theory of generalized inverses applied to robotics," Int. J. Robot. Res., vol. 12, no. 1, pp. 1-19, 1993.

[11] K. L. Doty, C. Melchiorri, E. M. Schwartz, and C. Bonivento, "Robot manipulability," IEEE Trans. Robot. Autom., vol. 11, no. 3, pp. 462-468, Jun. 1995

[12] M. Fujiwara, Y. Yokokohji, and T. Yoshikawa, "Guideline for designing haptic master hands based on dynamic muli-fingered manipulability," in Proc. 11th Symp. Haptic Interfaces Virtual Environ. Teleoperator Syst., 2003, pp. 77-84.

[13] S. Lee, "Dual redundant arm configuration optimization with task-oriented dual arm manipulability," IEEE Trans. Robot. Autom., vol. 5, no. 1, pp. 78-97, Feb. 1989

[14] Z. Li, P. Hsu, and S. Sastry, "Grasping and coordinated manipulation by a multifingered robot hand," Int. J. Robot. Res., vol. 8, no. 4, pp. 33-50, 1989.

[15] C. Melchiorri, "Comments on "global task space manipulability ellipsoids for multiple-arm systems" and further considerations," IEEE Trans. Robot. Autom., vol. 9, no. 2, pp. 232-236, Apr. 1993.

[16] J. San Martin and G. Trivino, "Mechanical design of a minimal invasive surgery trainer using the manipulability as measure of optimization," in Proc. IEEE Int. Conf. Mechatronics (ICM), Kumamoto, Japan, May 2007, pp. $1-5$.

[17] T. Yoshikawa, "Dynamic manipulability of robot manipulators," J. Robot. Syst., vol. 2, no. 1, pp. 113-124, 1985.
[18] T. Yoshikawa, "Virtual truss model for characterization of internal forces for multiple finger grasps," IEEE Trans. Robot. Autom., vol. 15, no. 5, pp. 941-947, Oct. 1999.

[19] T. Yoshikawa, "Erratum to "dynamic manipulability of robot manipulators"," J. Robot. Syst., vol. 17, no. 8, pp. 449-449, 2000.

[20] T. Yoshikawa, "Control algorithm for grasping and manipulation by multifingered robot hands using virtual truss model representation of internal force," in Proc. IEEE Int. Conf. Robot. Autom., 2000, pp. 369376

[21] X.-Z. Zheng, N. Tomochika, and T. Yoshikawa, "Dynamic manipulability of multiple robotic mechanisms in coordinated manipulation," in Proc. IFToMM-jc Int. Symp. Theory Mach. Mech., 1992, pp. 147-152.

[22] B.-R. Zuo and W.-H. Qian, "A general dynamic force distribution algorithm for multifingered grasping," IEEE Trans. Syst., Man, Cybern., vol. 30, no. 1, pp. 185-192, Feb. 2000

\section{Evaluation of Generalized Force Derivatives by Means of a Recursive Newton-Euler Approach}

\author{
Corrado Guarino Lo Bianco, Member, IEEE
}

\begin{abstract}
An accurate estimation of the dynamics efforts acting on a robot manipulator represents an important issue for both the analysis of its behavior and the synthesis of appropriate controllers. This paper proposes an iterative algorithm, which is based on the Newton-Euler approach, for the efficient evaluation of the manipulators' high-order kinematics and dynamics. In particular, the algorithm computes velocities, accelerations, and jerks of each link, while new dynamic equations are devised in order to evaluate the first derivative of generalized forces. Due to its moderate computational burden, the algorithm is suited to be used in online applications.
\end{abstract}

Index Terms-Dynamics, force derivative, jerk, kinematics, NewtonEuler, rigid-body manipulators.

\section{INTRODUCTION}

Manipulators' joint forces and torques, i.e., the generalized forces (GFs), are naturally bounded due to the actuators physical limits. For this reason, the GF bounding problem has been widely investigated in the past. Several solutions have been proposed, which can be roughly divided into two categories: offline and online approaches. Offline approaches are normally based on algorithms for the optimal trajectory planning that consider the existence of kinematic and/or dynamic constraints. For example, in [1], a minimum-time movement along an assigned path was planned by accounting for constraints on joint velocities and torques. However, when an optimal trajectory is used, there is at least one joint that is constantly working at the maximum of its kinematic or dynamic capabilities: Due to model uncertainties, the control could be lost easily. Several online algorithms have been developed in the past to handle this problem. The scheme that is originally proposed in [2] is often cited as an example: Dynamic constraints are satisfied by means of an online method that automatically and appropriately scales assigned trajectories.

Similarly, GF derivatives (GFDs) are also physically bounded. For example, in the case of electric actuators, the available supply voltage

Manuscript received September 8, 2008; revised February 9, 2009. First published July 6, 2009; current version published July 31, 2009. This paper was recommended for publication by Associate Editor A. Albu-Schäffer and Editor W. K. Chung upon evaluation of the reviewers' comments. This work was supported in part by the Automation, Electronics and Bioengineering: Technologies for Manufacturing and People (AER-TECH Lab), Emilia Romagna, Italy.

The author is with the Dipartimento di Ingegneria dell'Informazione, University of Parma, Parma I-43100, Italy (e-mail: guarino@ce.unipr.it).

Digital Object Identifier 10.1109/TRO.2009.2024787 